\title{
Embedded Flexible Force Sensor for In-Situ Tire-Road Interaction Measurements
}

\author{
Yizhai Zhang, Student Member, IEEE, Jingang Yi, Senior Member, IEEE, and Tao Liu, Member, IEEE
}

\begin{abstract}
In-situ sensing the tire-road interactions such as local contact friction force distributions provides crucial information for building accurate friction force models for vehicle safety control. In this paper, we report the development of an embedded, flexible local force sensor for measuring the tire local friction forces and their distributions. A new pressuresensitive, electric conductive rubber (PSECR) sensor is used and embedded inside the tire rubber layer to extract the multidimensional local friction forces on the tire contact patch. The low-cost, flexible PSECR sensor is oriented in certain directions, and is sensitive to multiple compressive forces. To interpret the sensor measurements, we use a beam-spring model for the tireroad interactions to extract the local contact friction forces. The experimental results of stick-slip interaction testing on a motorcycle tire demonstrate the effective and good performance of the PSECR-based tire force sensor.
\end{abstract}

Index Terms-Contact model, pneumatic tire, stick-slip interactions, tactile sensing, tire sensors.

\section{INTRODUCTION}

$\mathbf{P}$ NEUMATIC tires are widely used for ground vehicles. Tire-road interactions such as tire friction forces play an extremely important role for safe operation of these vehicles. Building high-fidelity tire-road friction models is crucial for vehicle dynamic simulations and active safety control [1]-[3]. Non-intrusive, in-situ sensing of the tire-road interactions provides direct information about the local force distributions on the tire contact patch and helps build friction force models [4]. It is however challenging to measure the local friction forces on the tire contact patch. It is a challenging task to develop a sensing system to measure the contact friction forces of deformable rubber tires. Due to the highly coupling effects between the longitudinal and lateral friction forces and the normal contact force, extracting and obtaining all 3-D forces simultaneously adds further modeling challenges to interpret sensor measurements.

Manuscript received November 3, 2012; revised December 25, 2012; accepted January 10, 2013. Date of publication January 18, 2013; date of current version April 2, 2013. This work was supported in part by the U.S National Science Foundation under Award CMMI-0856095. The associate editor coordinating the review of this paper and approving it for publication was Prof. Elena Gaura. (Corresponding author: J. Yi.)

Y. Zhang and J. Yi are with the Department of Mechanical and Aerospace Engineering, Rutgers University, Piscataway, NJ 08854 USA (e-mail: yzzhang@eden.rutgers.edu; jgyi@rutgers.edu).

T. Liu is with the Department of Intelligent Mechanical Systems Engineering, Kochi University of Technology, Kochi 782-8502, Japan (e-mail: liu.tao@kochi-tech.ac.jp).

Color versions of one or more of the figures in this paper are available online at http://ieeexplore.ieee.org.

Digital Object Identifier 10.1109/JSEN.2013.2241051
The US Congress TREAD Act requires the mandatory installation of a tire pressure monitoring system (TPMS) in all US automobiles and light trucks for safety reasons [5]. However, the TPMS is not designed for obtaining the friction forces. Tire rubber deformation and stresses contain the most direct information for understanding and modeling tire-road interaction forces [1], [3], [4]. Various tire sensors are developed to measure the rubber tread deformation and stresses. In [6], [7], a tire sensor has been developed to measure the tire tread deformation. There is however no further reported evaluation on tire-road friction forces from the measured tread deflection. Surface acoustic wave (SAW) sensors are proposed for the "smart tire" application in [8]. Strain and capacitance sensors have been developed for monitoring the tire tread deformation [9], [10]. In [11], [12], accelerometers are placed inside tires for friction force estimations. Optical sensors are also developed for measuring tire deformation [13]. The optical sensing system is complicated and might not be very robust for varying tire running environments and for realtime applications. Piezoelectric sensors such as polyvinylidene fluoride (PVDF) has been proposed and used for measuring the stresses on the inlet surface of the tire [14]. The stress measurements are interpreted through a friction force model. The results in [14] demonstrate the feasibility of the PVDFbased tread deformation sensor. In [15], a differently configured PVDF-based deformation sensor is designed to measure the tire sidewall deformation and then to estimate the tire slip angle. Built on these measurements, the tire lateral friction force is estimated using an analytical force model. A similar PVDF-based deformation sensor is also reported in [16].

Although the above discussed tire sensors provide measurements of the forces and the deformations, few results are reported to capture the local friction forces and their distributions. Moreover, these tire sensors cannot capture all aspects of the tire/road interaction forces. For example, the piezoelectric tire sensors such as PVDF cannot capture the static loading and forces. It is also challenging to use strain and capacitance sensors to measure multi-directional forces. In this paper, we present the development of a new pressure-sensitive, electric conductive rubber (PSECR) sensor to directly measure local friction forces during tire/road stickslip interaction. Comparing with the existing reported tire sensors, we choose the PSECR sensor to measure the local friction forces between the tire and the road because of its attractive properties such as multi-sensing cells within one small unit, flexibility, high sensitivity to both static and dynamic 


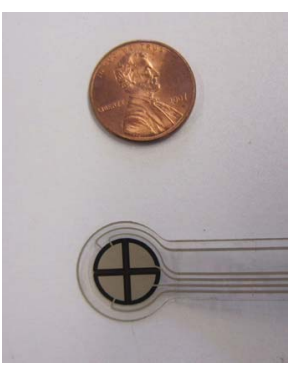

(a)

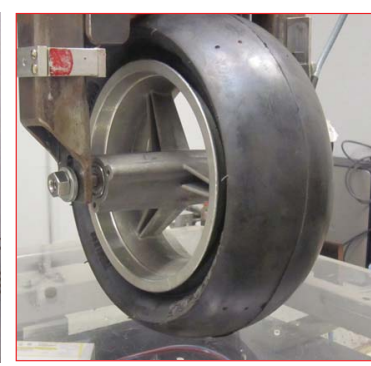

(b)

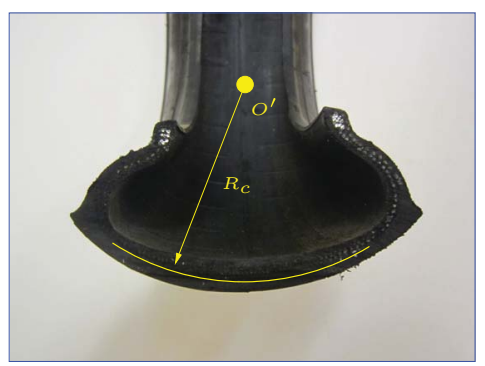

(c)

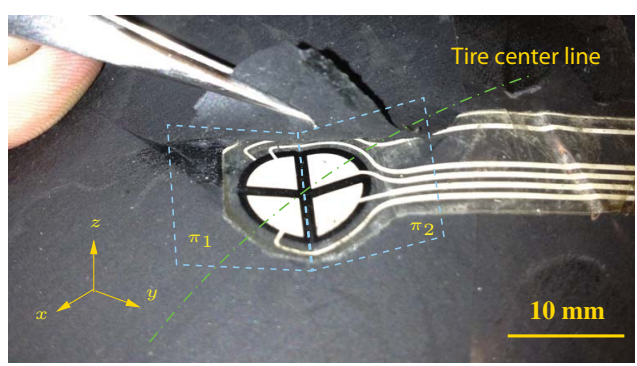

(d)

Fig. 1. (a) PSECR sensor. (b) Motorcycle tire used in experiments. (c) Cross section of the tire. (d) PSECR sensor embedded inside the tire.

forces, and low-cost [17]. To interpret the PSECR sensor measurements and obtain the local friction forces, we use and extend the beam-spring network model developed in [18] to build the sensor model. The sensor system development is also inspired by the fingertip tactile sensing for robotic manipulation applications [19]. We finally demonstrate the PSECR sensor performance through applications and experiments to study the local friction forces and their distributions during the stick-slip interactions between the stationary tire and the firm road. Measuring stick-slip interaction friction forces is used as the sensor application for several reasons. First, understanding the stick-slip interaction provides the foundation to capture the dynamic tire-road interactions. Second, obtaining the local friction forces and their distributions in stick-slip interactions is a challenging task due to large rubber deformation and complex normal load distributions, etc. Finally, although we present the sensor development for stick-slip friction force measurement, the sensor model can be extended to dynamic tire sensing.

The main contribution of this work lies in the new local force sensor prototype and modeling development for studying friction contact problems in tire-road interactions. The proposed sensor directly measures and captures the local friction forces. The local friction sensing model can also be potentially used for other mechanical systems in which a large deformation exists between a flexible thin layer and a rigid. To the best of our knowledge, there are no sensing, modeling and experimental studies that reveal the contact force distributions between the tire and the road, or in general a deformable thin layer and a rigid. The sensing system developed in this work fills the gap of such knowledge.

The rest of the paper is organized as follows. In Section II, we discuss the embedded PSECR sensor development. In Section III, we present the sensing model to extract the local friction forces. The PSECR sensor calibration and experiments are presented in Section IV. We conclude the paper in Section V.

\section{EMBEDDED TIRE SENSORS}

\section{A. PSECR Sensor}

The PSECR sensor (from Pongpara Codan Rubber Technology Company, Thailand) is shown in Fig. 1(a). The circular sensor is around $12-\mathrm{mm}$ in diameter and is flexible. In one sensor unit, there are four sensing cells that generate independent measurement outputs. The output voltage of each sensor cell is proportional to the compressive pressure perpendicularly applied on the sensor surface. Due to the thin-film packaging, the PSECR sensor cannot work under applied tensile stress. The details of the PSECR sensor are described in [17].

We use a motorcycle tire to embed the PSECR sensor. The motorcycle tire is shown in Fig. 1(b) and the tire has smooth outer surfaces. The cross-section of the tire has a circular shape as shown in Fig. 1(c). For the torus-shape tire, it is straightforward to obtain that the tire-road contact patch forms an elliptical shape [20]. We choose this type of motorcycle tires with simple geometry and structure such that we can focus on the fundamental mechanical contact properties without distraction by the influences of other factors such as tire treads and grooves etc. Although we choose this simple-geometry tire, as shown in experiments in this paper and in [18], the contact properties such as the normal contact pressure distribution follow the similar pattern as those obtained by using actual automobile tires reported in [21], [22].

The sensor is embedded at the center line of the contact patch; see Fig. 1(d). To minimize the destructive effects, cutting and gluing process is carefully conducted. The small cut is created using a scalpel. The $3 \mathrm{M}$ Scotch-Weld Neoprene high performance rubber glue is used to maintain the homogeneous rubber property within the cut area. As we will show in the later sections, the consistent comparison results between the sensor measurements and the model predictions confirm that the destructive effect on the tire structure due to cutting is negligible.

\section{B. "Smart Tire" Testing Platform}

Fig. 2 shows the "smart tire" testing platform. A treadmill is modified to act as a firm foundation to hold the tire support plate. To study tire-road stick-slip interactions for PSECR sensor testing, we replace the treadmill belt with a thick transparent plate. The plate is supported by three individually computer-controlled jacks. Three load cells (from Transducer Techniques Inc.) are positioned under the plate to measure the total normal load between the tire and the plate.

To generate friction forces along the $x$ - and $y$-axis directions, we use two steel cables to pull the tire: one applied on tire rim for the longitudinal ( $x$-axis) direction and the other applied on the transparent plate for the lateral ( $y$-axis) direction. The total friction forces along $x$ - and $y$-directions 


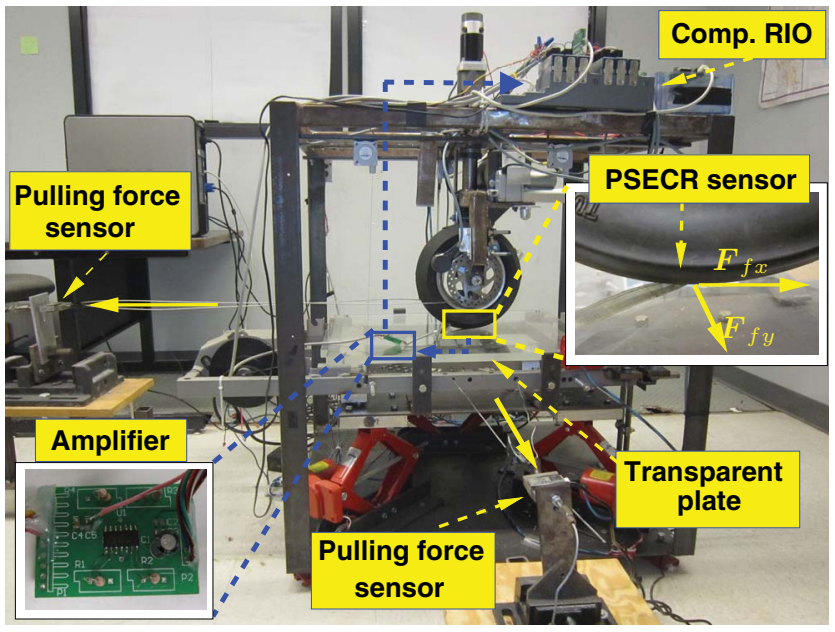

Fig. 2. "Smart tire" testing platform with an embedded force sensor inside the tire. We use force sensors to measure the total friction forces on the tire contact patch.

are respectively measured by two force sensors as shown in Fig. 2. The data collection and the normal force control are implemented through a real-time NI CompactRIO embedded system. The details about the setup are presented in [20].

\section{SENSOR MODELS}

In this section, we first briefly review the beam-spring model for the tire-road interactions. Then we present the model to interpret the sensor measurements and to extract the local friction forces on the tire contact patch. Finally, we discuss the optimal orientation of the embedded sensor inside the tire rubber layer.

\section{A. Beam-Spring Tire-Road Interaction Models}

Fig. 3(a) shows the schematic of the tire-road contact. We denote the contact patch as $\mathcal{P}$ and the thickness of the rubber layer as $2 h$. A coordinate system is set up with the origin at the center of $\mathcal{P}$ with a vertical distance of $h$ to the road surface. The $x$-axis is along the tire center with the positive direction towards the leading edge of $\mathcal{P}$, that is, the direction where the tire tends to move forward. We denote the total longitudinal and lateral friction forces as $F_{f x}$ and $F_{f y}$, respectively. The resultant of $F_{f x}$ and $F_{f y}$ is defined by $\mathbf{F}_{f}$. We denote the tire normal contact pressure distribution on $\mathcal{P}$ as $P_{n}(x, y)$ with the inflation air pressure $P_{\text {air }}$ and the total normal force $F_{z}$. We denote the friction force distribution at point $(x, y)$ on $\mathcal{P}$ as $\mathbf{P}_{f}(x, y)$ with the $x$ - and $y$-axis components as $P_{f x}(x, y)$ and $P_{f y}(x, y)$, respectively. Therefore, we have

$$
F_{z}=\int_{\mathcal{P}} P_{n}(x, y) d S, \quad \mathbf{F}_{f}=\int_{\mathcal{P}} \mathbf{P}_{f}(x, y) d S
$$

where $d S$ is an infinitesimal area on $\mathcal{P}$.

For stationary tire-road contact, when total friction force $\mathbf{F}_{f}$ increases, the contact goes through a stick-to-slip transition [18]. The stick contact refers to no locally relative movement between the tire and the road, while in slip contact, local relative tire-road motion exists. During the stick-to-slip transition, some contact points at certain locations on $\mathcal{P}$ begin

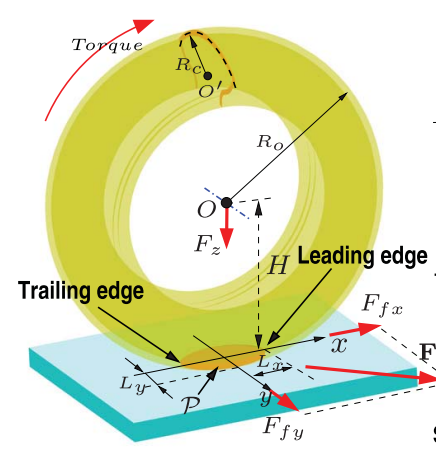

(a)

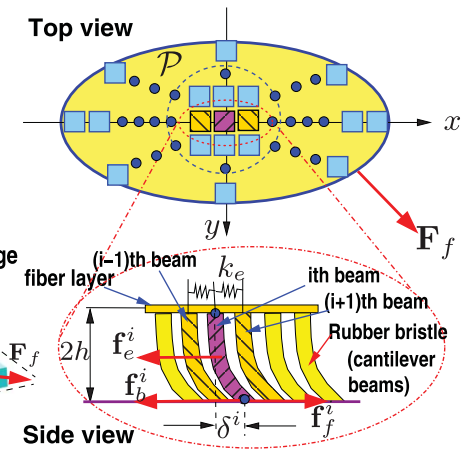

(b)
Fig. 3. (a) Schematic diagram of the tire-road contact patch. (b) Schematic diagram of the beam-spring network model for stick-slip interactions.

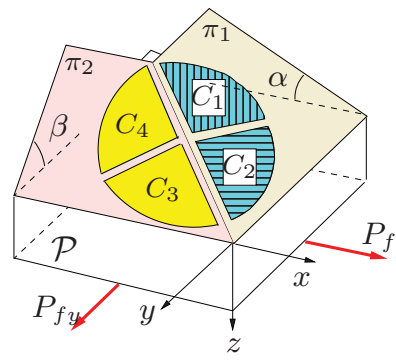

(a)

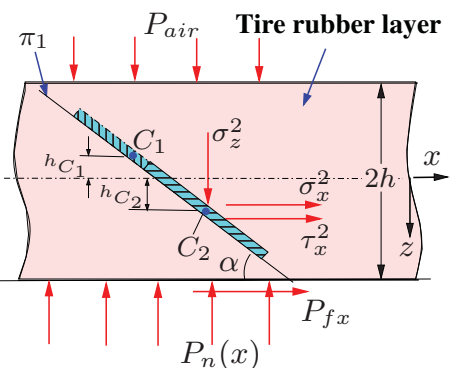

(b)
Fig. 4. Embedded PSECR sensor configuration. (a) Configuration diagrams of the four sensor cells. (b) Side view schematic diagram of the embedded sensor inside the rubber layer.

deforming and slipping while other points remain sticking to the road. The slipping region grows as the applied force increases. We use a beam-spring network modeling approach to capture the stick-slip interactions.

Fig. 3(b) illustrates the beam-spring network modeling approach. We partition the tire-road contact into $N$ virtual cantilever beams and each of them has a height of $2 h$ and a square cross-section. Linear springs are assumed to connect each pair of neighboring beams. During the stick-to-slip transition, dynamic motions of the beams are negligible. For the $i$ th beam inside the interior of $\mathcal{P}, i=1, \ldots, N$, the following force equilibrium is obtained.

$$
\mathbf{f}_{f}^{i}+\mathbf{f}_{b}^{i}+\mathbf{f}_{e}^{i}=0
$$

where $\mathbf{f}_{f}^{i}$ is the tire contact friction force, $\mathbf{f}_{b}{ }^{i}$ is the bending force that captures the shear deformation, and $\mathbf{f}_{e}^{i}$ is the resultant net elastic force from the adjacent beams. In [18], we have discussed how to obtain $\mathbf{f}_{b}{ }^{i}$ and $\mathbf{f}_{e}^{i}$ such that we can then use (1) to calculate $\mathbf{f}_{f}^{i}$.

In the following, we describe how to use the beam-spring network model to extract the friction forces on $\mathcal{P}$ from the measured forces by the PSECR sensor inside the tire rubber.

\section{B. PSECR Sensor Output Model}

To obtain the multi-directional local friction forces, the sensor is embedded with a special orientation as shown in Fig. 4. If being positioned horizontally, the PSECR sensor is not sensitive to local friction forces on $\mathcal{P}$. Moreover, the PSECR sensor only functions under compressive forces inside 
the rubber layer and thus, it cannot be placed along the vertical direction since horizontal tensile stresses exist during the stickto-slip transition. By tilting the sensor with a certain angle, the existence of the internal compressive normal stress prevents the sensor from exposing to tensile forces.

To extract both the local $x$ - and $y$-axis directional friction forces, we separate the four sensor cells into two groups: $C_{1}$ and $C_{2}$ are placed on plane $\pi_{1}$, while $C_{3}$ and $C_{4}$ lie in plane $\pi_{2}$. The tilting angles of $\pi_{1}$ and $\pi_{2}$ with respect to the $x$ - and $y$-axis directions are denoted as $\alpha$ and $\beta$, respectively. We will discuss how to determine the optimal values for $\alpha$ and $\beta$ in Section III-C.

Let $P_{f x}$ and $P_{f y}$ denote the local friction stresses at location $(x, y)$ on $\mathcal{P}$ along the $x$ - and $y$-axis directions at the sensor location, respectively; see Fig. 4(a). $P_{f x}$ and $P_{f y}$ can be considered as the $x$ - and $y$-axis components of the spatially normalized friction force $\mathbf{f}_{f}^{i}$ given in the beam-spring network model in (1); see Fig. 3(b). Due to symmetry of $\mathcal{P}$ and for presentation clarity, we consider locations on $\mathcal{P}$ along the tire center line (e.g., at $y=0$ ) and varying $x$ coordinate so that the force variables are only functions of $x$. Let $\sigma_{k}^{l}$ and $\tau_{k}^{l}$ denote the normal and shear stresses along the $k$-axis for $C_{l}$, $k=x, y, l=1,2,3,4$, respectively. Note that $\sigma_{k}^{l}$ corresponds to the spring elasticity effect and is considered as the spatially normalized spring force in the beam-spring network model. Similarly, $\tau_{k}^{l}$ is related to the bending effect at the sensor location. We denote the normal stress along the $z$-direction at the sensor location as $\sigma_{z}^{l}$.

Let $F_{s}^{l}$ denote the resultant compressive force applied to $C_{l}$ and $V_{s}^{l}$ be its measured output voltage. Then we have $V_{s}^{l}=$ $K_{l} F_{s}^{l}$, where $K_{l}, l=1,2,3,4$, are the output gains for sensor cells $C_{l}$ [17]. The sensor output $V_{s}$ under $P_{f x}$ and $P_{f y}$ consists of two portions: one portion $V_{0}$ is due to the rubber bending and the other portion comes from the existence of $\sigma_{k}^{l}$ and $\tau_{k}^{l}$ due to the friction forces on $\mathcal{P}$. Thus, output $V_{s}^{l}$ can be written as

$$
V_{s}^{l}= \begin{cases}V_{0}^{l}+K_{l} A_{s}\left(-\sigma_{x}^{l}+\tau_{x}^{l}\right) \sin \alpha, & l=1,2, \\ V_{0}^{l}+K_{l} A_{s}\left(-\sigma_{y}^{l}+\tau_{y}^{l}\right) \sin \beta, & l=3,4,\end{cases}
$$

where

$$
V_{0}^{l}= \begin{cases}K_{l} A_{s}\left(-\sigma_{z}^{l} \cos \alpha-\sigma_{x 0}^{l} \sin \alpha\right), & l=1,2, \\ K_{l} A_{s}\left(-\sigma_{z}^{l} \cos \beta-\sigma_{y 0}^{l} \sin \beta\right), & l=3,4,\end{cases}
$$

where $A_{s}$ is the area of each sensor cell, and $\sigma_{x 0}$ and $\sigma_{y 0}$ are the shell-to-plate bending stresses along the $x$ - and $y$-axis, respectively.

The goal of building a sensor model is to extract $P_{f x}$ and $P_{f y}$ information from measurements $V_{s}^{l}$ in (2). We first obtain a model for $V_{0}^{l}$. By the assumption of the unchanged length of neutral line during the shell-to-plate bending [23] and considering the thin rubber layer, we estimate

$$
\sigma_{x 0}^{l} \approx-\frac{E h_{C_{l}}}{R_{o}-h}, l=1,2, \quad \text { and } \quad \sigma_{y 0}^{l} \approx-\frac{E h_{C_{l}}}{R_{c}-h}, l=3,4 \text {, }
$$

where $h_{C_{l}}$ is the $z$ coordinate of cell $C_{l}$. To obtain $\sigma_{z}(z)$, we obtain the following relationship

$$
\sigma_{z}(z)=\left[P_{n}(x)-P_{a i r}\right]\left(\frac{1}{4 h^{3}} z^{3}-\frac{3}{4 h} z\right)-\frac{P_{n}(x)+P_{a i r}}{2},
$$

where $P_{n}(x)$ is the contact normal pressure and can be estimated empirically [20]. The derivation of the above equation is given in Appendix I. Combining the above results for $\sigma_{x 0}^{l}$, $\sigma_{y 0}^{l}$ and $\sigma_{z}^{l}=\sigma_{z}\left(h_{C_{l}}\right)$ and from (3), we obtain $V_{0}^{l}$ in (2).

We need to build the relationship between $\sigma_{k}^{l}$, $\tau_{k}^{l}$ and $P_{f x}, P_{f y}$. In the following discussion, we only present the development of such relationships for $C_{1}$ and $C_{2}$ to extract $P_{f x}$ and the similar results can be obtained along the $y$-axis direction for $C_{3}$ and $C_{4}$ to obtain $P_{f y}$.

We denote the spatially normalized net spring force $\mathbf{f}_{e}^{i}$ and beam bending force $\mathbf{f}_{b}^{i}$ along the $x$-axis direction as $\sigma_{\mathcal{P} x}$ and $\tau_{\mathcal{P} x}$, respectively. We normalize (1) spatially, take the magnitude of the normalized stress equation along the $x$-axis direction, and then obtain

$$
P_{f x}=\tau_{\mathcal{P} x}+\sigma_{\mathcal{P} x} .
$$

Notice that $\tau_{\mathcal{P} x}$ corresponds to the shear stress $\tau_{x}^{l}$ and $\sigma_{\mathcal{P} x}$ is related to the normal stress $\sigma_{x}^{l}$. Both $\sigma_{x}^{l}$ and $\tau_{x}^{l}$ can be extracted from the sensor measurements through (2) for $C_{1}$ and $C_{2}$. First, the sensor cells are spatially close each other and in the local area of $C_{1}$ and $C_{2}, \sigma_{x}^{l}, l=1,2$, are treated approximately the same because of the small sensor size. Therefore, we have

$$
\sigma_{x}^{1}=\sigma_{x}^{2}=\sigma_{x} .
$$

To further build the relationship between $\tau_{x}^{l}$ and $\tau_{\mathcal{P}_{x}}$, we consider that $\tau_{\mathcal{P} x}$ is the boundary shear stress at $z=h$ and $\tau_{x}^{l}$, $l=1,2$, are the shear stresses at the sensor locations $z_{l}=h_{C_{l}}$. We assume that the shear deflection of the rubber layer follows the virtual beam deflection. Using the Euler-Bernoulli beam theory, the deflection $w(z)$ of a cantilever beam under bending force $f_{b x}$ is

$$
w(z)=\frac{f_{b x}(z+h)^{2}(5 h-z)}{6 E I}
$$

and the (maximal) deflection at the tip is $\delta_{x}=\frac{f_{b x}(2 h)^{3}}{3 E I}$. By eliminating $f_{b x}$, we obtain

$$
w(z)=\frac{\delta_{x}}{16 h^{3}}\left(-z^{3}+3 h z^{2}+9 h^{2} z+5 h^{3}\right)
$$

and $\tau_{x}^{l}$ is then calculated by

$$
\tau_{x}^{l}=\left.G \frac{\partial w(z)}{\partial z}\right|_{z=h_{C_{l}}}=\left.\gamma_{l} G \frac{\partial w(z)}{\partial z}\right|_{z=h}=\gamma_{l} \tau_{\mathcal{P} x},
$$

where $\gamma_{l}=\frac{1}{4 h^{2}}\left(-h_{C_{l}}^{2}+2 h_{C_{l}} h+3 h^{2}\right), l=1$, 2. Eq. (7) implies that $\tau_{x}^{l}$ varies along the $z$-direction.

Using (2), (6) and (7), we obtain

$$
\underbrace{\left[\begin{array}{c}
V_{s}^{1}-V_{0}^{1} \\
V_{s}^{2}-V_{0}^{2}
\end{array}\right]}_{\mathbf{V}}=\underbrace{\left[\begin{array}{ll}
-K_{1} A_{s} \sin \alpha & K_{1} A_{s} \gamma_{1} \sin \alpha \\
-K_{2} A_{s} \sin \alpha & K_{2} A_{s} \gamma_{2} \sin \alpha
\end{array}\right]}_{\mathbf{K}} \underbrace{\left[\begin{array}{c}
\sigma_{x} \\
\tau_{\mathcal{P}}
\end{array}\right]}_{\sigma} .
$$

The coefficient matrix $\mathbf{K}$ is invertible if $\gamma_{1} \neq \gamma_{2}$, that is, $C_{1}$ and $C_{2}$ are not at the same depth, which is satisfied in the design. $\sigma_{x}$ and $\tau_{\mathcal{P} x}$ are then uniquely determined as $\sigma=$ $\mathbf{K}^{-1} \mathbf{V}$. The linear model in (8) is derived based on the EulerBernoulli beam assumption and other modeling approaches might lead to the similar conclusion. Instead computing $\mathbf{K}$ in (8) from a set of the model parameters, we conduct a calibration process to obtain $\mathbf{K}$ in practice for high accuracy. 
Therefore, the final results of obtaining coefficient $\mathbf{K}$ are not restricted by knowing the beam model parameters.

We still need to obtain $\sigma_{\mathcal{P} x}$ to calculate $P_{f x}$ by (5). We take an empirical approach to calculate $\sigma_{\mathcal{P}_{x}}$ : if the sensor lies in the stick region, we take the following relationship

$$
\sigma_{\mathcal{P} x}=\kappa_{x}\left|\sigma_{x}\right|^{m_{x}},
$$

where we use the absolute value of $\sigma_{x}$ because $\sigma_{x}$ can be negative and $\sigma_{\mathcal{P}_{x}}$ is always positive. The coefficients $\kappa_{x}$ and $m_{x}$ are obtained by calibration in experiments. When the sensor lies in the location where the local contact slips, $\sigma_{\mathcal{P} x}$ saturates at the value $\sigma_{\mathcal{P}_{x}}^{0}$. We detect slipping when $\tau_{\mathcal{P} x}$ is saturated and at this moment, we denote $\sigma_{x}=\sigma_{x}^{0}$. Then $\sigma_{\mathcal{P} x}^{0}$ is calculated by (9) for the given $\sigma_{x}^{0}$.

Summarizing the above discussion, we obtain

$$
P_{f x}= \begin{cases}\tau_{\mathcal{P} x}+\sigma_{\mathcal{P} x} & \text { locally stick, } \\ \tau_{\mathcal{P} x}+\sigma_{\mathcal{P} x}^{0} & \text { locally slip }\end{cases}
$$

where $\tau_{\mathcal{P} x}$ and $\sigma_{\mathcal{P} x}$ are respectively obtained by (8) and (9) with a calibrated PSECR sensor, and $\sigma_{\mathcal{P} x}^{0}=\kappa_{x}\left|\sigma_{x}^{0}\right|^{m_{x}}$ with $\sigma_{x}^{0}$ is captured by sensor measurements as discussed above.

\section{Optimal Sensor Orientation}

The tilting angles $\alpha$ and $\beta$ of the PSECR sensor can be optimized for enhancing force measurements. Due to the symmetry, we discuss how to obtain the optimal value for $\alpha$ and the similar conclusion is applicable to obtain the optimal value for $\beta$.

From (8), angle $\alpha$ should be maintained as large as possible to have a superior sensitivity and resolution for stresses $\sigma$. Furthermore, from the first equation in (3), a large $\alpha$ also helps minimize the influences of the variations of $V_{0}^{l}$ in measurements due to the small $\sigma_{x 0}^{l}$ term. On the other hand, the PSECR sensor only works under compressive stresses and a large $\alpha$ potentially risks elongation stresses applied to the sensor because of the possible tensile stress distribution inside the rubber layer (see Fig. 12(a)). We thus enforce the constraint $V_{s}^{l} \geq 0$ to guarantee always compressive forces on the sensor. With the above considerations, we formulate the following optimization problem:

$$
\begin{array}{ll}
\operatorname{maximize} & \alpha \\
\text { subject to } & -\sigma_{z}^{l} \cos \alpha+\left(-\sigma_{x}^{l}+\tau_{x}^{l}\right) \sin \alpha \geq 0 \\
& 0 \leq \alpha \leq 90^{\circ}, \quad l=1,2
\end{array}
$$

The constraint (11b) is obtained by plugging (2) into $V_{s}^{l} \geq 0$ and neglecting the term $\sigma_{x 0}^{l}$ because of its negligible value.

The constraint should be satisfied for all possible stresses $\sigma_{z}^{l}$, $\sigma_{x}^{l}$ and $\tau_{x}^{l}$ on $\mathcal{P}$. We consider the fact that $\sigma_{z}^{l}<0$ and the only possibility of having elongation stresses on the sensor exists when $-\sigma_{x}^{l}+\tau_{x}^{l}<0$. Therefore, we solve (11) by considering the case of $\tau_{x}^{l}<\sigma_{x}^{l}$, and obtain

$$
\alpha_{\max }=\min _{l}\left\{\min \tan ^{-1}\left(\frac{-\sigma_{z}^{l}}{\sigma_{x}^{l}-\tau_{x}^{l}}\right)\right\}, \quad \tau_{x}^{l}<\sigma_{x}^{l}
$$

for $l=1,2$. In the above equation, we take the minimal value of all possible cases of $\tau_{x}^{l}<\sigma_{x}^{l}$ for both $C_{1}$ and $C_{2}$.

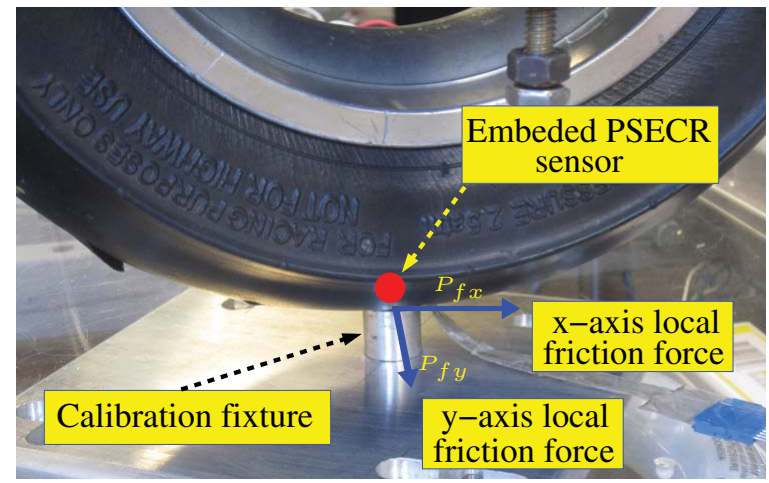

Fig. 5. PSECR sensor calibration fixture.

To illustrate a typical value of $\alpha_{\max }$, we consider a case of $P_{a i r}=69 \mathrm{kPa}$ and $F_{z}=267 \mathrm{~N}$ (used in experiments), and obtain $\alpha_{\max }=41$ degs. Due to the small size of the PSECR sensor and the thin rubber layer thickness $(2 h=7 \mathrm{~mm})$, we cannot install the PSECR sensor at $\alpha_{\max }=41$ degs. Instead, $\alpha=21$ degs and $\beta=26$ degs are the largest possible values that satisfy (11) and that are obtained to embed the PSECR sensor.

\section{EXPERIMENTS}

In this section, we present the experiments to calibrate the PSECR sensor, validate the sensor models, and demonstrate the sensor performance through an example of the tire-road stick-slip interactions.

\section{A. PSECR Sensor Calibration and Model Validation}

The sensor calibration processes for the $x$ - and $y$-direction forces are similar and we here only describe the $x$-direction case. We first obtain the $\sigma_{x}$ measurements right after the sensor is glued on one side inside the tire rubber. We use a force gauge (model FDK 80 from Wagner Inc.) to press on $C_{1}$ and $C_{2}$ to generate $\sigma_{x}$. Fig. 6(a) shows the outputs of $C_{1}$ and $C_{2}$ under various $\sigma_{x}$ measurements. The sensor outputs demonstrate a strong linear relationship with $\sigma_{x}$.

After the sensor is completely glued inside the tire, we apply $\tau_{\mathcal{P} x}$ to the local area where the PSECR sensor is embedded using a calibration fixture. The fixture is designed and fabricated with a comparable size to that of the PSECR sensor [20]; see Fig. 5. The fixture is directly mounted on the transparent plate of the testing platform (Fig. 2). The use of the calibration fixture guarantees that the friction force lie locally at the location where the sensor is embedded. We put a leather cover on the fixture surface to generate high friction forces and prevent the contact from sliding. Therefore, shear force dominates the interaction and we assume that the local friction force $P_{f x}$ is fully contributed by $\tau_{\mathcal{P} x}$. The calibration results with varying $\tau_{\mathcal{P}_{x}}$ are shown in Fig. 6(b). A linear relationship between the sensor outputs and $\tau_{\mathcal{P} x}$ is clearly obtained, similar to the model prediction by (7). Moreover, the results also show that the two sensor cells have different sensitivities to $\tau_{\mathcal{P} x}$, as the model (7) predicts due to the different $\gamma_{l}$ at locations of $C_{l}, l=1,2$. 


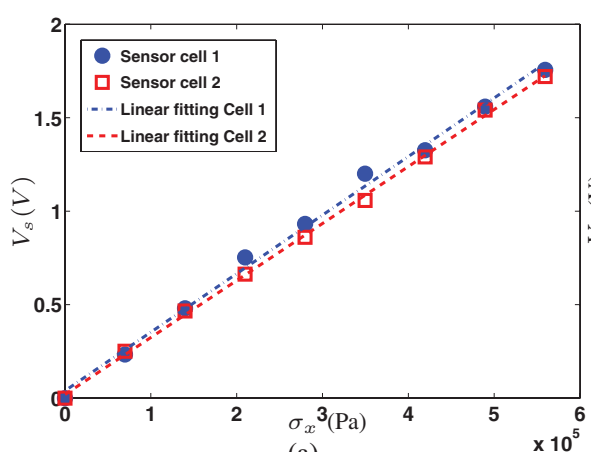

(a)

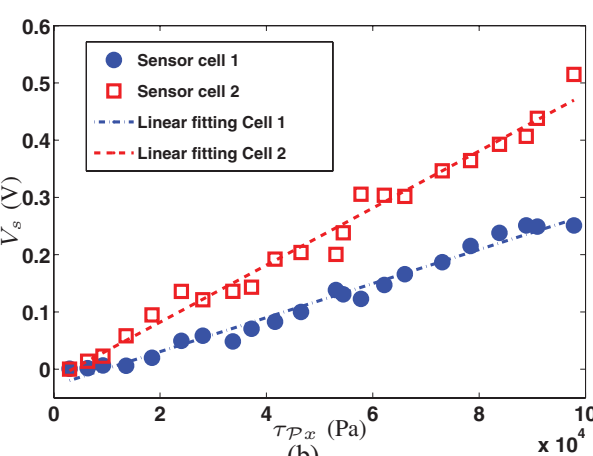

(b)

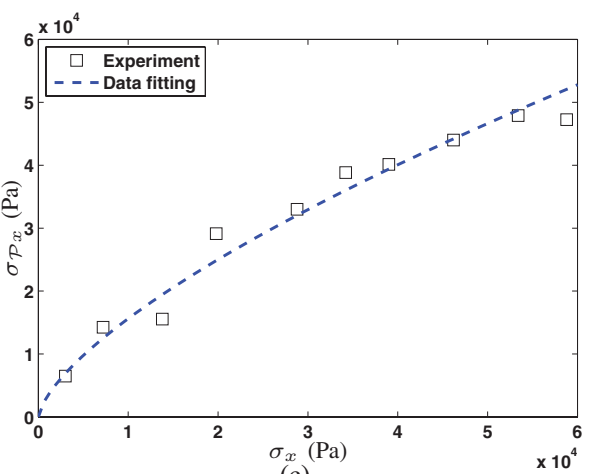

(c)

Fig. 6. Sensor cells $C_{1}$ and $C_{2}$ calibration results. (a) Sensor output voltage with various $\sigma_{x}$. (b) Sensor output voltage with various $\tau_{\mathcal{P}}$. (c) Gain $\kappa_{x}$ (between $\sigma_{\mathcal{P} x}$ and $\sigma_{x}$ ) calibration.

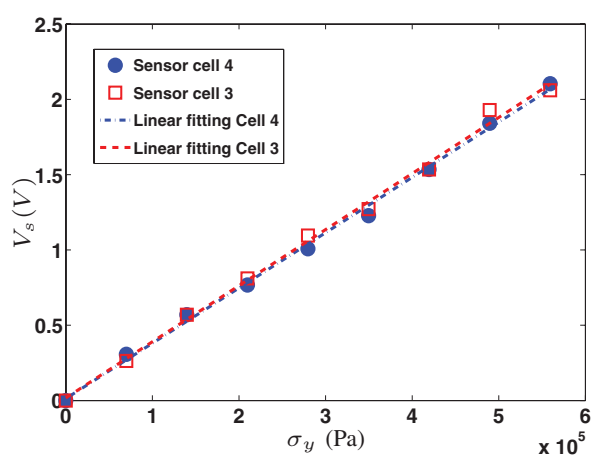

(a)

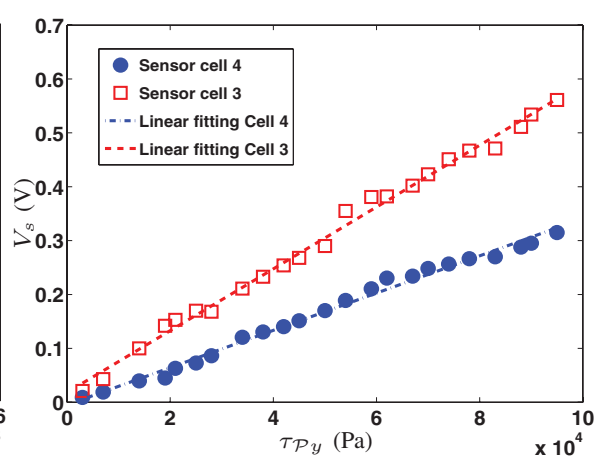

(b)

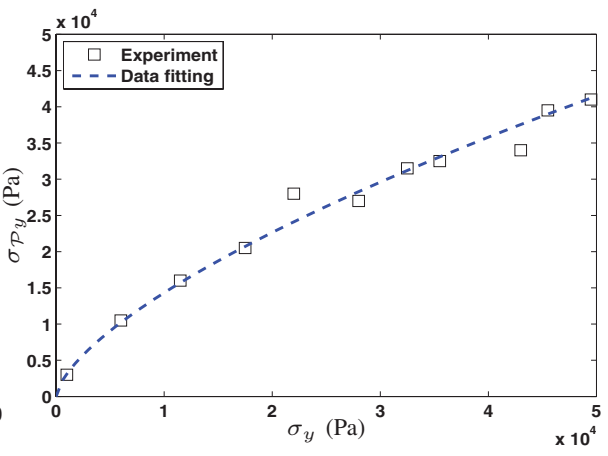

(c)

Fig. 7. Sensor cells $C_{3}$ and $C_{4}$ calibration results. (a) Sensor output voltage with various $\sigma_{y}$. (b) Sensor output voltage with various $\tau_{\mathcal{P}}$. (c) Gain $\kappa_{y}$ (between $\sigma_{\mathcal{P} y}$ and $\sigma_{y}$ ) calibration.

In experiments, multiple repeated calibrations are conducted. Given $M_{1}$ measurements of $\sigma_{x}=\left(\sigma_{x}\right)_{M_{1}}$ and $M_{2}$ measurements of $\boldsymbol{\tau}_{\mathcal{P}_{x}}=\left(\tau_{\mathcal{P}_{x}}\right)_{M_{2}}$, we form a stress measurement matrix $\sigma_{m} \in \mathbb{R}^{2 \times\left(M_{1}+M_{2}\right)}$ and the corresponding sensor output matrix $\mathbf{V}_{m} \in \mathbb{R}^{2 \times\left(M_{1}+M_{2}\right)}$ respectively as

$$
\boldsymbol{\sigma}_{\boldsymbol{m}}=\left[\begin{array}{cc}
\boldsymbol{\sigma}_{x} & \mathbf{0}_{M_{2}} \\
\mathbf{0}_{M_{1}} & \boldsymbol{\tau}_{\mathcal{P} x}
\end{array}\right] \text { and } \mathbf{V}_{m}=\left[\begin{array}{c}
V_{s}^{1}-V_{0}^{1} \\
V_{s}^{2}-V_{0}^{2}
\end{array}\right],
$$

where $\mathbf{0}_{M} \in \mathbb{R}^{1 \times M}$ is the zero row vector. From the relationship $\mathbf{V}=\mathbf{K} \sigma_{\boldsymbol{m}}$ in (8), we estimate $\mathbf{K}$ in the $x$-direction as

$$
\hat{\mathbf{K}}_{x}=\mathbf{V}_{m} \boldsymbol{\sigma}_{m}^{+}=\mathbf{V}_{m} \boldsymbol{\sigma}_{m}^{T}\left(\boldsymbol{\sigma}_{m} \boldsymbol{\sigma}_{m}^{T}\right)^{-1},
$$

where $\boldsymbol{\sigma}_{m}^{+}=\boldsymbol{\sigma}_{m}^{T}\left(\boldsymbol{\sigma}_{m} \boldsymbol{\sigma}_{m}^{T}\right)^{-1}$ is the pseudo-inverse of $\boldsymbol{\sigma}_{m}$. The mean and the standard deviation values of the calibrated $\mathbf{K}_{x}$ are respectively as

$$
\hat{\mathbf{K}}_{x}=\left[\begin{array}{ll}
-3.01 & 2.73 \\
-3.02 & 4.84
\end{array}\right] \mathrm{V} / \mathrm{MPa}
$$

and

$$
\hat{\sigma}_{\mathbf{K}_{x}}=\left[\begin{array}{ll}
0.09 & 0.18 \\
0.10 & 0.23
\end{array}\right] \mathrm{V} / \mathrm{MPa} .
$$

By substituting $\hat{\mathbf{K}}_{x}$ into the sensor output model, we then calibrate coefficients $\kappa_{x}$ and $m_{x}$ in (9) with measurements $\sigma_{x}$ and $\sigma_{\mathcal{P} x}$, where $\sigma_{\mathcal{P} x}=P_{f x}-\tau_{\mathcal{P} x}$ and $P_{f x}$ is obtained again from the calibration fixture without the leather cover; see Fig. 5. Fig. 6(c) shows the calibration curve. The calibration values of estimated $\kappa_{x}$ and $m_{x}$ are $\hat{\kappa}_{x}=29.74$ and $\hat{m}_{x}=0.68$, respectively. The standard deviations of the estimated $\kappa_{x}$ and $m_{x}$ are $\hat{\sigma}_{\kappa_{x}}=1.97$ and $\hat{\sigma}_{m_{x}}=0.01$, respectively.

A similar calibration process is conducted for the $y$ direction force sensor cells $C_{3}$ and $C_{4}$. The calibration results are shown in Fig. 7 and the calibration coefficients in the $y$ direction are

$$
\hat{\mathbf{K}}_{y}=\left[\begin{array}{ll}
-3.32 & 2.97 \\
-3.33 & 5.03
\end{array}\right] \mathrm{V} / \mathrm{MPa}, \hat{\kappa}_{y}=32.40 \text { and } \hat{m}_{y}=0.66
$$

respectively. The standard deviations for the estimated $\mathbf{K}_{y}, \kappa_{y}$ and $m_{y}$ are respectively as

$$
\hat{\sigma}_{\mathbf{K}_{y}}=\left[\begin{array}{ll}
0.13 & 0.22 \\
0.11 & 0.24
\end{array}\right] \mathrm{V} / \mathrm{MPa}, \hat{\sigma}_{\kappa_{y}}=1.91 \text { and } \hat{\sigma}_{m_{y}}=0.01 \text {. }
$$

To validate the sensor model, we conduct multiple repeated validation tests by varying $P_{f x}$ and $P_{f y}$ at the sensor location and then compare with the sensor measurements. Fig. 8 shows the comparison results for both $P_{f x}$ and $P_{f y}$ in one validation test. The root mean square differences are $1.1 \times 10^{4} \mathrm{~Pa}$ (for the $x$-direction stress) and $0.9 \times 10^{4} \mathrm{~Pa}$ (for the $y$-direction stress). Fig. 9 shows the statistical errors of the multiple validation tests. The maximum errors are consistently less than $1.5 \times 10^{4} \mathrm{~Pa}$ in both the $x$ - and $y$-direction. We also compare the measured voltage $V_{0}$ with the model prediction (3) as shown in Fig. 10. The values for the $V_{0}$ model parameters are listed in Table I. The model predictions show a clear agreement with the experiments for $V_{0}$. 


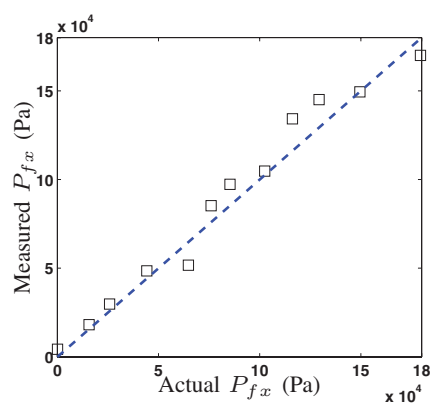

(a)

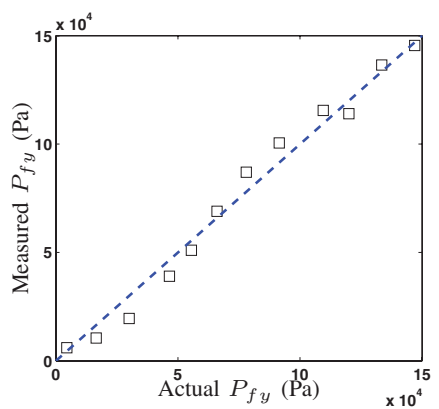

(b)
Fig. 8. Friction force measurement validations. (a) $P_{f x}$ measurements. (b) $P_{f y}$ measurements.
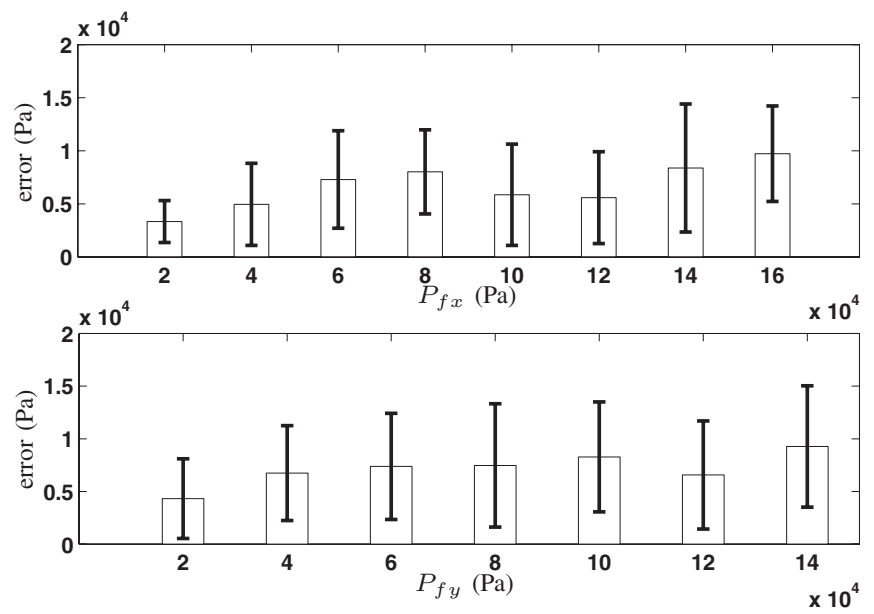

Fig. 9. Statistical mean errors and their standard deviations of sensor measurements.

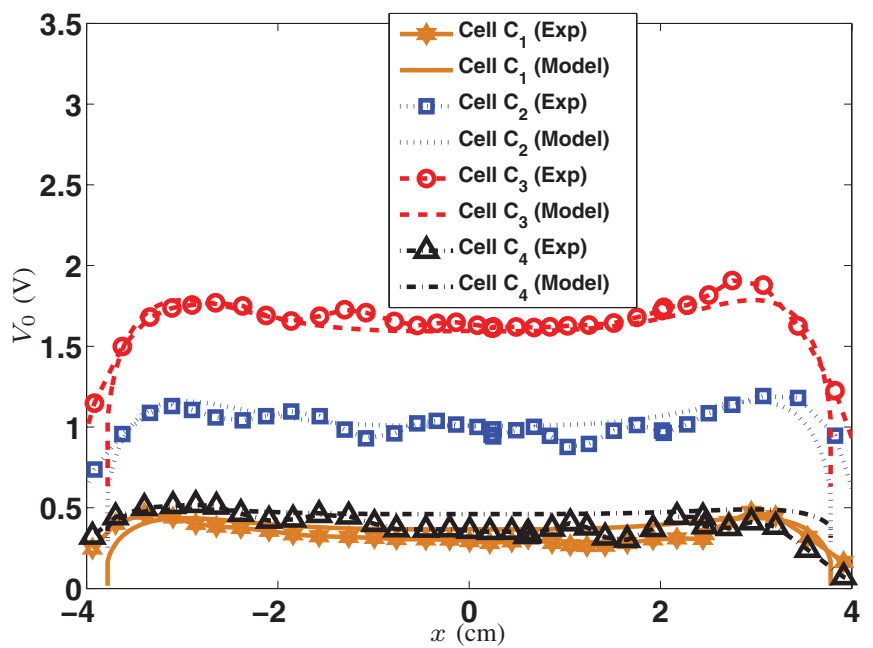

Fig. 10. Comparison of the prediction of the static bending stress (represented by $V_{0}$ ) with the experiments.

\section{B. Friction Force Distributions in Stick-to-Slip Transition}

We use the calibrated PSECR sensor to measure the friction force distribution on $\mathcal{P}$ during the stick-to-slip transition. We compare the experiments with the prediction results from the model in [18] to validate the sensor measurements and demonstrate the sensor performance.

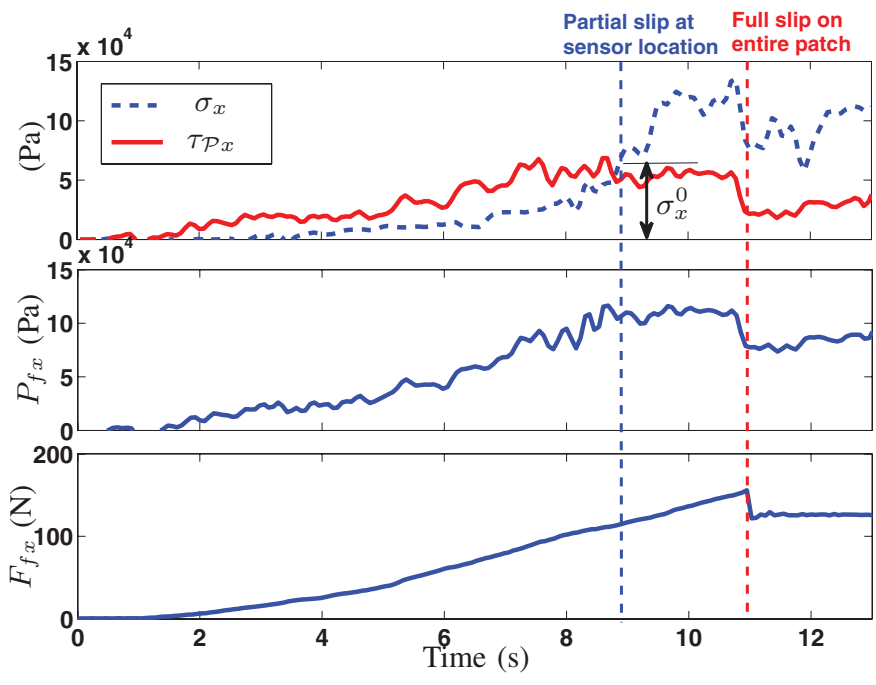

Fig. 11. PSECR sensor measurements and stress estimation at the sensor location during the stick-to-slip transition.

TABLE I

PSECR SENSOR AND TIRE PARAMETERS

\begin{tabular}{|c|c|c|c|c|c|c|c|}
\hline$E(\mathrm{MPa})$ & $R_{O}(\mathrm{~m})$ & $h(\mathrm{~mm})$ & $A_{S}\left(\mathrm{~cm}^{2}\right)$ & $K_{1}(\mathrm{~V} / \mathrm{N})$ & $K_{2}(\mathrm{~V} / \mathrm{N})$ & $K_{3}(\mathrm{~V} / \mathrm{N})$ & $K_{4}(\mathrm{~V} / \mathrm{N})$ \\
\hline 11.7 & 0.128 & 3.5 & 0.16 & 0.56 & 0.54 & 0.53 & 0.53 \\
\hline
\end{tabular}

To observe the stick-to-slip transition and the local friction force evolutions for a point on $\mathcal{P}$, we rotate the tire to locate the sensor around the middle point between the trailing edge and the center of $\mathcal{P}$ (Fig. 2). An increasing $F_{f x}$ is generated by pulling the cable until the tire completely slips at around $F_{f x}=160 \mathrm{~N}$. Fig. 11 shows the sensor measurements at the local area where the sensor is embedded. Shear stress $\tau_{\mathcal{P} x}$ starts to increase with the increasing $F_{f x}$. Once the partial slip at the sensor location starts around $t=8.8 \mathrm{~s}, \tau_{\mathcal{P} x}$ stops increasing and keeps flat. This observation agrees with the friction model in which $P_{f x}$ saturates when partial slip starts. The observation of $\tau_{\mathcal{P} x}$ becoming flat is used to predict the moment when the partial slip happens at the sensor location. We estimate $\sigma_{x}^{0}=6 \times 10^{4} \mathrm{~Pa}$ and therefore, $\sigma_{\mathcal{P} x}^{0}=\hat{\kappa}_{x}\left|\sigma_{x}^{0}\right|^{\hat{m}_{x}}=$ $5.76 \times 10^{4} \mathrm{~Pa}$.

After the partial slip starts, $\sigma_{x}$ keeps increasing with the increasing $F_{f x}$ and the slip region propagates towards the interior portion of $\mathcal{P}$. $P_{f x}$ shown in the middle plot of Fig. 11 is the local friction force measurement during the stick-to-slip transition. When the full slip of the entire patch starts around $t=11 \mathrm{~s}, \sigma_{x}, \tau_{\mathcal{P}_{x}}$ and $P_{f x}$ drop rapidly.

To illustrate $\sigma_{x}(x)$ and $P_{f x}(x)$ distributions under $F_{f x}$, we conduct a set of scanning experiments by moving the embedded sensors at various locations along the tire center line (i.e., $y=0$ ), while pulling the tire until completely slipping. Fig. 12(a) demonstrates the comparison results between the experiments and the model predictions for local stress $\sigma_{x}(x)$. The results show a clear agreement between the sensor measurements and the model calculations. The results in Fig. 12(a) show that $\sigma_{x}(x)$ has larger values close to the edge of $\mathcal{P}$ than those around the center of $\mathcal{P}$. Notice the sign change of $\sigma_{x}(x)$, at the leading edge the rubber is under compression 

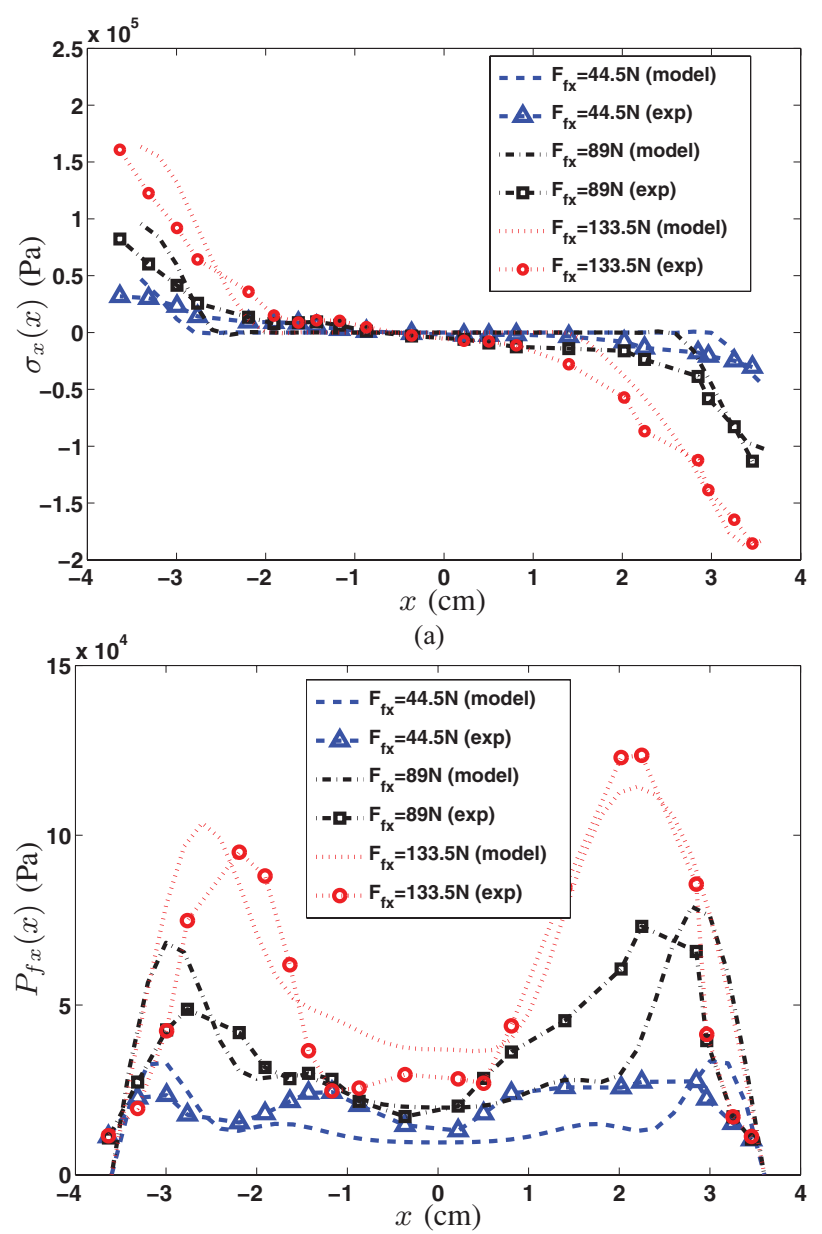

(b)

Fig. 12. Comparison results between the sensor measurements of stress distributions and the model prediction on $\mathcal{P}$ under various friction forces $F_{f x}$. (a) $\sigma_{x}(x)$ and (b) $P_{f x}(x)$.

(i.e., negative $\sigma_{x}(x)$ ), while at the trailing edge portion it is under elongation (i.e., positive $\sigma_{x}(x)$ ). This phenomenon also matches the rubber deformation distribution reported in [18]. Fig. 12(b) shows the local friction stress $P_{f x}(x)$ along the center line of $\mathcal{P}$. Again, the sensor measurements fit the numerical calculations.

Fig. 13 shows the consistent matching results between the experiments and the model predictions for $P_{f y}(x)$ along the center line of $\mathcal{P}$ under lateral force $F_{f y}$. Similar to $P_{f x}(x)$, the sensor measurements of $P_{f y}(x)$ match the model prediction. We also conduct experiments under the existence of both $F_{f x}$ and $F_{f y}$ and the results consistently show the agreements between the sensor measurements and the model predictions. These results confirm the capability of the PSECR sensor to capture the local friction forces and their distributions on $\mathcal{P}$.

Some discrepancies exist between the sensor measurements and the model predictions. These differences mainly come from several sources. Due to the physical constraints, the sensor is not installed at the optimal orientation for superior sensitivity. The imperfect numerical model calculations also contribute partially to the discrepancies. The complex rubber hyper-elastic property also results in sensor prediction errors. The accuracy limitation of the low-cost PSECR sensor is another source of the measurement errors.

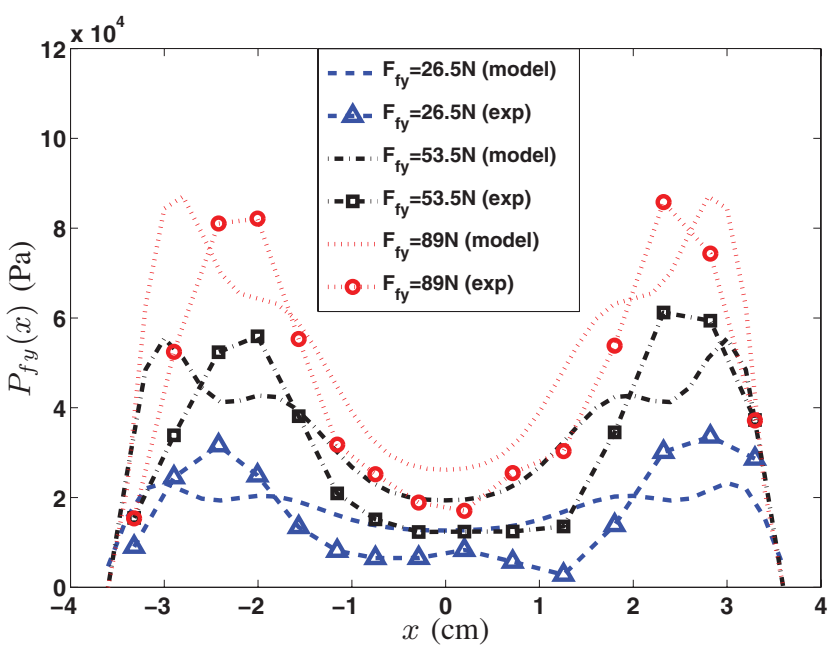

Fig. 13. Comparison results of the sensor measurements and the model prediction for $P_{f y}(x)$ distribution under applying various friction forces $F_{f y}$ along the center line.

\section{CONClusion}

We presented a new, flexible force sensor to capture the in-situ tire local friction force distributions in stick-slip transition. The flexible force sensor is made of the pressure sensitive, electric conductive rubber (PSECR). The PSECR sensor has four independent sensing cells within one unit and these spatially distributed sensor arrays enabled us to extract the multi-dimensional local friction forces simultaneously. We analyzed and interpreted the PSECR sensor measurements by using the beam-spring network model. The sensor calibration process and results were presented and the performance was also demonstrated through experiments to extract the local friction forces in tire-road stick-slip interactions. The sensor measurements showed high agreements with the numerical results for the friction force distributions. The PSECR sensor has the potentials to capture local friction forces in contact between a flexible thin layer and a rigid in other applications.

\section{APPENDIX I}

\section{Calculation of $\sigma_{z}(z)$ Inside The Rubber Layer}

Fig. 4(b) illustrates the configuration of the tire rubber layer. We first decompose $P_{n}(x)$ by a Fourier series.

$$
P_{n}(x)=P_{0}+\sum_{m=1}^{\infty} A_{m} \sin (\lambda x)+\sum_{n=1}^{\infty} B_{m} \cos (\lambda x),
$$

where $P_{0}$ is the constant term and $\lambda=\frac{m \pi}{L_{x}}$ and $L_{x}$ is the major axis length of the elliptical $\mathcal{P}$. By superposition, we consider the contribution of each term in (14) to the internal stress $\sigma_{z}(z)$. The particular solution of $\sigma_{z}(z)$ when $P_{\text {air }}$ and $P_{0}$ are applied on each side of the tire rubber layer is given by (page 41 of [23])

$$
\sigma_{z}^{s}(z)=\frac{P_{0}-P_{a i r}}{4 h^{3}} z^{3}-\frac{3\left(P_{0}-P_{a i r}\right)}{4 h} z-\frac{P_{0}+P_{\text {air }}}{2} .
$$

To calculate the contribution of the first harmonic term $A_{m} \sin (\lambda x)$ in (14) to $\sigma_{z}(z)$, we introduce an Airy function $\phi_{m}(x, z)=\sin (\lambda x) f_{m}(z)$ in the form of the Fourier 
series [23]. Plugging $\phi_{m}(x, z)$ into bi-harmonic condition $\nabla^{4} \phi_{m}(x, z)=0$, we obtain

$$
\lambda^{4} f_{m}(z)-2 \lambda^{2} f_{m}^{\prime \prime}(z)+f_{m}^{(4)}(z)=0 .
$$

The general solution for $f_{m}(z)$ is

$$
f_{m}(z)=D_{1} \operatorname{ch}(\lambda z)+D_{2} \operatorname{sh}(\lambda z)+D_{3 z} \operatorname{ch}(\lambda z)+D_{4 z} \operatorname{sh}(\lambda z)
$$

where $\operatorname{sh}(x):=\sinh (x)$ and $\operatorname{ch}(x):=\cosh (x)$ for $x$.

Considering that $\sigma_{z}=\frac{\partial^{2} \phi_{m}}{\partial x^{2}}$ and $\tau_{x z}=\frac{\partial^{2} \phi_{m}}{\partial x \partial z}$ and the boundary conditions: $\tau_{x z}=0, \sigma_{z}=-A_{m} \sin \lambda x$ for $z=h$, and $\tau_{x z}=0$ and $\sigma_{z}=0$ for $z=-h$, we obtain coefficients $D_{i}$ in (15). Therefore, the contribution of the term $A_{m} \sin (\lambda x)$ in (14) for $P_{n}(x)$ is given by $\sigma_{s}^{m}$ as

$$
\begin{aligned}
& \sigma_{s}^{m}=-A_{m} \sin (\lambda x) \\
& \quad \times\left\{\frac{[\lambda h \operatorname{ch}(\lambda h)+\operatorname{sh}(\lambda h)] \operatorname{ch}(\lambda z)-\lambda z \operatorname{sh}(\lambda z) \operatorname{sh}(\lambda h)}{\operatorname{sh}(2 \lambda h)+2 \lambda h}\right. \\
& \left.+\frac{[\lambda h \operatorname{sh}(\lambda h)+\operatorname{ch}(\lambda h)] \operatorname{sh}(\lambda z)-\lambda z \operatorname{ch}(\lambda z) \cos (\lambda h)}{\operatorname{sh}(2 \lambda h)-2 \lambda h}\right\} .
\end{aligned}
$$

Similarly, for the term $B_{m} \cos (\lambda x)$ in (14) for $P_{n}(x)$, the contribution to the stress distribution is given by $\sigma_{c}^{m}$ as

$$
\begin{aligned}
& \sigma_{c}^{m}=-B_{m} \cos (\lambda x) \\
& \quad \times\left\{\frac{[\lambda h \operatorname{ch}(\lambda h)+\operatorname{sh}(\lambda h)] \operatorname{ch}(\lambda z)-\lambda z \operatorname{sh}(\lambda z) \operatorname{sh}(\lambda h)}{\operatorname{sh}(2 \lambda h)+2 \lambda h}\right. \\
& \left.+\frac{[\lambda h \operatorname{sh}(\lambda h)+\operatorname{ch}(\lambda h)] \operatorname{sh}(\lambda z)-\lambda z \operatorname{ch}(\lambda z) \cos (\lambda h)}{\operatorname{sh}(2 \lambda h)-2 \lambda h}\right\} .
\end{aligned}
$$

For $L_{x} \gg h$, using the approximations $\operatorname{sh}(x) \approx x+\frac{x^{3}}{6}+\frac{x^{5}}{120}$ and $\operatorname{ch}(x) \approx 1+\frac{x^{2}}{2}+\frac{x^{4}}{24}$ for small $x$, Eqs. (16) and (17) are reduced respectively to

$$
\begin{aligned}
\sigma_{s}^{m} & \approx A_{m} \sin (\lambda x)\left(\frac{1}{4 h^{3}} z^{3}-\frac{3}{4 h} z-\frac{1}{2}\right) \text { and } \\
\sigma_{c}^{m} & \approx B_{m} \cos (\lambda x)\left(\frac{1}{4 h^{3}} z^{3}-\frac{3}{4 h} z-\frac{1}{2}\right) .
\end{aligned}
$$

By superposition, we add all the above calculated stress terms together and using (14), we obtain

$$
\begin{aligned}
\sigma_{z}(z) & =\sigma_{z}^{s}(z)+\sum_{m=1}^{\infty} \sigma_{s}^{m}+\sum_{m=1}^{\infty} \sigma_{c}^{m} \\
& =\left[P_{n}(x)-P_{\text {air }}\right]\left(\frac{1}{4 h^{3}} z^{3}-\frac{3}{4 h} z\right)-\frac{P_{n}(x)+P_{\text {air }}}{2}
\end{aligned}
$$

for a given $x$. This completes the calculation of (4). The above derivation is readily extended to the two-dimensional case (i.e., including stress along the $y$-axis direction) if $L_{y} \gg h$.

\section{ACKNOWLEDGMENTS}

The authors would like to thank Prof. L. Liu of Rutgers University for various helpful discussions. We are also grateful to A. Allen and the Rutgers "smart tire" senior design team in 2009 for designing, fabricating, and improving the original testing platform.

\section{REFERENCES}

[1] S. Müller, M. Uchanski, and K. Hedrick, "Estimation of the maximum tire-road friction coefficient," ASME J. Dynamic Syst., Meas., Control, vol. 125, no. 4, pp. 607-617, 2003.

[2] J. Yi, L. Alvarez, and R. Horowitz, "Adaptive emergency brake control with underestimation of friction coefficient," IEEE Trans. Control Syst. Technol., vol. 10, no. 3, pp. 381-392, May 2002.

[3] J. Li, Y. Zhang, and J. Yi, "A hybrid physical/dynamic tire/road friction model," ASME J. Dynamic Syst., Meas., Control, vol. 135, no. 1, pp. 011007-1-011007-11, Jan. 2013.

[4] Intelligent Tyre Systems - State of the Art and Potential Technologies, Technical Research Centre of Finland (VTT), Espoo, Finland, 2003.

[5] "Federal motor vehicle safety standard: Tire pressure monitoring systems, controls, and displays," NHTSA, Washington, DC, USA, Tech. Rep. 2000-8572, 2000.

[6] O. Yilmazoglu, M. Brandt, J. Sigmund, E. Genc, and H. Hartnagel, "Integrated InAs/GaSb 3-D magnetic field sensors for 'the intelligent tire'," Sens. Actuators, A, vol. 94, nos. 1-2, pp. 59-63, Oct. 2001.

[7] S. Gruber, M. Semsch, T. Strothjohann, and B. Breuer, "Elements of a mechatronic vehicle corner," Mechatronics, vol. 12, no. 8, pp. 1069-1080, 2002

[8] A. Pohl, R. Steindl, and L. Reindl, "The "Intelligent tire" utilizing passive saw sensors-measurement of tire friction," IEEE Trans. Instrum. Meas., vol. 48, no. 6, pp. 1041-1046, Dec. 1999.

[9] R. Matsuzaki and A. Todoroki, "Passive wireless strain monitoring of actual tire using capacitance-resistance change and multiple spectral features," Sens. Actuators, A, vol. 126, no. 2, pp. 277-286, Feb. 2006.

[10] R. Matsuzaki and A. Todoroki, "Wireless flexible capacitive sensor based on ultra-flexible epoxy resin for strain measurement of automobile tires," Sens. Actuators, A, vol. 140, no. 1, pp. 32-42, Oct. 2007.

[11] F. Braghin, M. Brusarosco, F. Cheli, A. Cigada, S. Manzoni, and F. Mancosu, "Measurement of contact forces and patch features by means of accelerometers fixed inside the tire to improve future car active control," Veh. Syst. Dyn., vol. 44, no. 1, pp. 3-13, 2006.

[12] S. Savaresi, M. Tanelli, P. Langthaler, and L. Del Re, "New regressors for the direct identification of tire deformation in road vehicles via "intire' accelerometers," IEEE Trans. Control Syst. Technol., vol. 16, no. 4, pp. 769-780, Jul. 2008.

[13] M. Palmer, C. C. Boyd, J. McManus, and S. Meller, "Wireless smarttires for road friction measurement and self state determination," in Proc. 43rd AIAA/ASME/ASCE/AHS Struct., Struct. Dynamics, Mater. Conf., Denver, CO, USA, Apr. 2002, no. AIAA2002-1548.

[14] J. Yi, "A piezo-sensor based 'smart tire' system for mobile robots and vehicles," IEEE/ASME Trans. Mechatron., vol. 13, no. 1, pp. 95-103, Feb. 2008.

[15] G. Erdogan, L. Alexander, and R. Rajamani, "Estimation of tire-road friction coefficient using a novel wireless piezoelectric tire sensor," IEEE Sensors J., vol. 11, no. 2, pp. 267-279, Feb. 2011.

[16] J. Yi and H. Liang, "A PVDF-based deformation and motion sensor: Modeling and experiments," IEEE Sensors J., vol. 8, no. 4 pp. 384-391, Apr. 2008.

[17] T. Liu, Y. Inoue, and K. Shibata, "A small and low-cost 3-D tactile sensor for a wearable force plate," IEEE Sensors J., vol. 9, no. 9, pp. 1103-1110, Sep. 2009.

[18] Y. Zhang and J. Yi, "Tire/road stick-slip interactions: Analysis and experiments," IEEE/ASME Trans. Mech., 2012, to be published.

[19] V.-A. Ho, D. V. Dao, and S. Hirai, "Development and analysis of a sliding tactile soft fingertip embedded with a microforce/moment sensor," IEEE Trans. Robot., vol. 27, no. 3, pp. 411-424, Jun. 2011.

[20] Y. Zhang, A. W. Allen, J. Yi, and T. Liu, "Understanding tire/road stick-slip interactions with embedded rubber force sensors," in Proc. IEEE/ASME Int. Conf. Adv. Intell. Mechatron., Jul. 2012, pp. 550-555.

[21] H. Shiobara, T. Akasaka, and S. Kagami, "Two-dimensional contact pressure distribution of a radial tire in motion," Tire Sci. Technol., vol. 24, no. 4, pp. 294-320, 1996.

[22] S. Kim, P. Nikravesh, and G. Gim, "A two-dimensional tire model on uneven roads for vehicle dynamic simulations," Veh. Syst. Dyn., vol. 46, no. 10, pp. 913-930, 2008.

[23] S. P. Timoshenko, Theory of Elasticity. New York, USA: McGraw-Hill, 1970. 


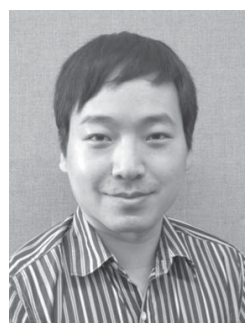

Yizhai Zhang (S'11) received the B.S. degree and the M.S. degree in information and communication engineering from Xi' an Jiaotong University, China, in 2005 and 2009, respectively. He is currently pursuing the Ph.D. degree in mechanical and aerospace engineering at Rutgers University, Piscataway, NJ, USA. His research interests include autonomous robotic systems, dynamic systems and control, intelligent sensing and actuation systems, and mechatronics.

Mr. Zhang is a student member of American Society of Mechanical Engineers (ASME). He received the Best Student Paper at the 2012 IEEE/ASME International Conference on Advanced Intelligent Mechatronics and the 2012 ASME Dynamic Systems and Control Conference.

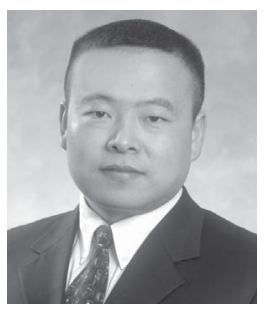

Jingang Yi (S'99-M'02-SM'07) received the B.S. degree in electrical engineering from Zhejiang University, Hangzhou, China, in 1993, the M.Eng. degree in precision instruments from Tsinghua University, Beijing, China, in 1996, and the M.A. degree in mathematics and the Ph.D. degree in mechanical engineering from the University of California, Berkeley, in 2001 and 2002, respectively. He is currently an Assistant Professor of Mechanical Engineering at Rutgers University. His research interests include autonomous robotic systems, dynamic systems and control, mechatronics, automation science and engineering, with applications to biomedical systems, civil infrastructure and transportation systems.
Dr. Yi is a member of American Society of Mechanical Engineers (ASME). $\mathrm{He}$ is a recipient of the 2010 US NSF CAREER Award. He has co-authored papers that have been awarded the Best Student Paper at the 2012 IEEE/ASME International Conference on Advanced Intelligent Mechatronics and the 2012 ASME Dynamic Systems and Control Conference, the Kayamori Best Automation Paper at the 2005 IEEE International Conference on Robotics and Automation, and several Best Paper finalists at the 2007 and 2008 IEEE International Conference on Automation Science and Engineering and the 2008 ASME Dynamic Systems and Control Conference. Dr. Yi currently serves as an Associate Editor for the IEEE TRANSACTIONS ON AUTOMATION SCIENCE AND ENGINEERING and the IEEE Robotics and Automation Society Conference Editorial Board (since 2008). He also served as a Guest Editor for the IEEE TRANSACTIONS ON AUTOMATION SCIENCE AND ENGINEERING in 2009 and an Associate Editor for the ASME Dynamic Systems and Control Division Conference Editorial Board from 2008 to 2010.

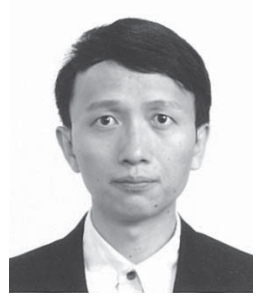

Tao Liu (M'08) received the M. Eng. degrees in mechanical engineering from the Harbin Institute of Technology, Harbin, China, in 2003 and the Doctorate degree in engineering from Kochi University of Technology, Kochi, Japan, in 2006. From 2003 to 2006, he was a Research Assistant at the Kochi University of Technology, where during 2007, he was a Postdoctoral Fellow in the Department of intelligent Mechanical Systems Engineering. He has been an Assistant Professor in the Department of Intelligent Mechanical Systems Engineering, since 2008. He was a recipient of the Japan Society of Mechanical Engineers Encouragement Prize (2010).

Dr Liu is also an inventor of one Japan patent about wearable sensors for gait analysis, which was commercialized. He has been serving as Editorial Board Member and Reviewer of multiple international journals, and serving as Program Committee member and Session Chair of multiple international conferences. His current research interests include wearable sensor systems, rehabilitation robots, biomechanics, and human motion analysis. 